\title{
EDITORIAL
}

\section{Theoretical Modeling and Simulation of Nanostructures}

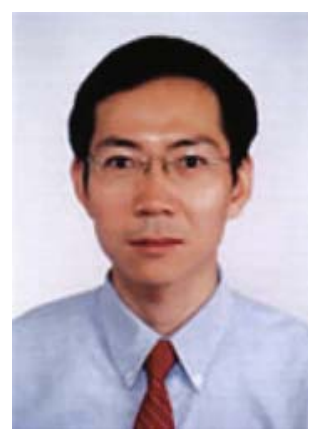

Prof. Jin-long YANG

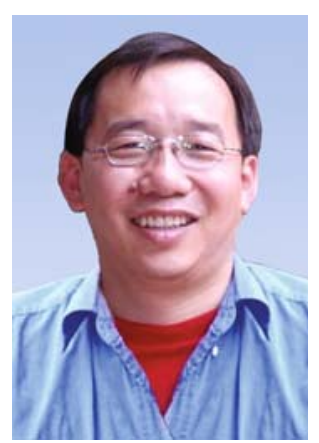

Prof. Yi LUO
In the recent decade, we have witnessed tremendous advances in nanoscience and nano-technology that promise to have a great impact on the future of information technology, clean energy, health care, and environments. Understanding the fundamentals of nanoscale properties holds the key to controlling and designing functional nano-material and nano-devices. At the nanoscale, quantum effects become dominant and the properties of nanomaterials and nano-devices are extremely sensitive to changes in their chemical and electronic structures. Experience and intuition are often no longer sufficient to provide reasonable interpretation for experimental observations. In this context, theoretical modeling plays a vital role in gaining insights. However, it is still a great challenge for theory to accurately describe nanostructures because of their size and peculiar many-body effects. Conventional computational methods can effectively treat small molecules and periodic systems with great accuracy, but still have difficulties applying to many finite systems at nanoscale because of the involvement of a large number of electrons. The confined electrons in nanoscale are strongly correlated, which can only be dealt with by density functional theory for the time being. It should always be remembered that none of the functionals available is perfect for every property. It is important to choose the right functional for the right purpose and to systematically improve the quality of the functionals in the future.

Theoretical modeling has actually been quite successful for some nano-materials and nano-devices, particularly nano-particles, nano-ribbons, nanotubes, and molecular electronic devices. One purpose of this special issue is thus to highlight these success stories. We have collected 20 articles in this special issue that give us a flavor of the current state-of-the-art in this field. They include review articles that summarize some of the recent methodology developments and applications in different areas, original research articles that present theoretical predictions of new functional materials, and verifications of experimental observations.

Finally, we thank all the authors and referees involved in this special issue for their contributions.

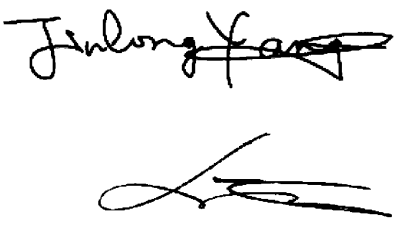

Guest Editors 